\title{
Low dose intrathecal morphine for hip arthroplasty
}

\section{Dosis baja de morfina intratecal para artroplastía de cadera}

Andrés Rojas G. MD, MSc. ${ }^{10,3}$, Marcela Hernández P. MD. ${ }^{1,3}$, Paola Vidal D. MD. ${ }^{2,3}$, Marco Balkenhol N. MD. ${ }^{1 a, 3}$, Marcela Opazo V. MD. ${ }^{1 b}$, Miguel Riquelme A. MD. ${ }^{1 c}$

\begin{abstract}
Objetives: $100 \mathrm{mcg}$ intrathecal morphine (ITM) for hip arthroplasty provides adequate functional recovery and reduces associated complications but is not exempt from opioid-related adverse effects. We evaluate efficacy of a reduced dose of ITM (80 mcg) in terms of anesthetic quality, postoperative analgesia, complication rates and early recovery. Methods: Case-control study. Patients under hip arthroplasty were treated on a specific protocol, using neuraxial anesthesia with hyperbaric bupivacaine $10.5-13.5 \mathrm{mg}$ plus $80 \mathrm{mcg}$ ITM versus controls with $100 \mathrm{mcg}$ ITM. Demographic variables, intra and perioperative course were extracted from medical records. Pain severity and morphine associated complications were blindly assessed at regular intervals postoperatively. $p$ $<0.01$ were considered significant. Results: 82 patients were analyzed. Mean age was 64.21 years, $62.20 \%$ women and $70.73 \%$ ASA-2. Main endoprosthesis indication was arthrosis (58.53\%). No statistically significant differences in demographic and operative data were found between groups, including surgical time, ambulation time, length of stay, and patient satisfaction for pain management. Mean VAS for pain during first 24 hours was 0.24 for the low ITM group and 0.22 for control. Rescue intravenous morphine was the same between groups. Compared to $80 \mathrm{mcg}$ ITM, $100 \mathrm{mcg}$ showed trends for higher complication rates for respiratory depression (OR 2.58, CI 95\% 0.45-14.54, $\mathrm{p}=$ 0.28 ), nausea without vomiting (OR $1.82, \mathrm{CI} 95 \% 0.82-4.01, \mathrm{p}=0.13$ ), urinary retention (OR 2.02, $\mathrm{Cl} 95 \% 0.88-4.61, \mathrm{p}=0.09)$ and significantly higher rates of pruritus (OR 3.55, Cl 95\% 1.61-7.82, p < 0.01). Conclusions: 80 mcg ITM
\end{abstract}

\section{Key words:}

Spinal anesthesia, intrathecal morphine, hip prosthesis, hip arthroplasty

\footnotetext{
Eduardo Schütz Schroeder Hospital. Puerto Montt, Chile.

Gustavo Fricke Hospital, Viña del Mar. Chile.

School of Medicine, San Sebastian University.

Department of Anesthesiology,

Department of Internal Medicine.

Department of Traumatology.
}

Fecha de recepción: 20 de noviembre de 2019

Fecha de aceptación: 25 de noviembre de 2019

\section{ORCID}

https://orcid.org/0000-0002-0634-1442

Corresponding author:

Andrés Rojas González

Email: andresirijillo@gmail.com 
during spinal anesthesia for hip arthroplasty provided comparable postoperative analgesia and lower incidence of opioid-related adverse effects.

\section{RESUMEN}

Objetivos: 100 mcg morfina intratecal (ITM), en artroplastia de cadera, proporciona una recuperación funcional adecuada y reduce complicaciones asociadas, pero no está exento de efectos adversos conocidos asociados a opioides. Evaluamos eficacia de reducir dosis ( $80 \mathrm{mcg}$ ITM) en términos de calidad anestésica, analgesia, complicaciones y recuperación postoperatoria. Métodos: Estudio de casos y controles. Pacientes sometidos a artroplastia de cadera fueron tratados con anestesia espinal con bupivacaína hiperbárica 10,5-13,5 mg más 80 mcg ITM y controles de manera similar pero con 100 mcg ITM. Variables demográficas, así como intra y perioperatorio, se extrajeron de registros médicos. Severidad del dolor, y complicaciones asociadas a ITM, se evaluaron a ciegas según protocolo, $\mathrm{p}<0,01$ fue considerado significativo. Resultados: 82 pacientes analizados. Edad promedio fue 64,21 años, 62,20\% fueron mujeres y $70,73 \%$ ASA-2. Principal indicación de prótesis fue artrosis (58,53\%). No se encontraron diferencias estadísticas entre variables demográficas, tiempo quirúrgico, tiempo deambulación, duración hospitalización y satisfacción paciente. EVA promedio dolor, primeras 24 horas, fue 0,24 para grupo 80 mcg ITM y 0,22 para control (100 mcg ITM). Morfina intravenosa de rescate fue similar entre grupos. En comparación con $80 \mathrm{mcg}, 100 \mathrm{mcg}$ presentó mayores tasas de complicaciones para depresión respiratoria (OR 2,58, IC95\% 0,45-14,54, p $=0,28)$, náuseas y vómitos ( $O R \quad 1,82, C 195 \% 0,82-4,01, p=0,13)$, retención urinaria $(\mathrm{OR} 2,02, \mathrm{C} 195 \% 0,88-4,61, \mathrm{p}=0,09)$ y prurito (OR 3,55, C195\% 1,61$7,82, p<0,01)$. Conclusiones: 80 mcg ITM, en anestesia espinal para artroplastia cadera, proporciona analgesia postoperatoria comparable a $100 \mathrm{mcg}$ pero con menor incidencia de efectos adversos relacionados a opioides.

\author{
Palabras clave: \\ Anestesia espinal, \\ morfina intratecal, \\ prótesis cadera, \\ artroplastia cadera
}

\section{Introduction}

P roviding adequate perioperative analgesia in patients undergoing partial or total hip arthroplasty requires careful evaluation and strict management by the treatment team, due to the increasing age and complexity of the patients and introduction of new anesthetic and surgical techniques. It is wellknown that instrumentation of the hip causes severe pain, especially in the first 24 hours[1]. Optimal pain management not only ensures patient comfort but also aids in functional recovery and reduces immediate and future complications[1],[2].

In recent years, ultrasound guided nerve blocks have been under development for pain managing in hip arthroplasty, but the use remain limited due to the need for equipment and technical proficiency[3],[4]. Meanwhile, multiple studies have demon- strated the benefits of intrathecal morphine in hip arthroplasty[3]-[5]. The consensus dose accepted at the current standard of care appears to be $100 \mathrm{mcg}$ of morphine, administered with a local anesthetic in a single-shot spinal block[1],[3]. However, patients often report breakthrough pain and opioid-related side effects such as pruritus and respiratory depression with this regimen[1]-[4]. Therefore, we aimed to evaluate the efficacy of a reduced dose of intrathecal morphine. We examined the anesthetic quality, postoperative analgesia, complication rates and functional recovery in patients who received low versus conventional dose ITM. We hypothesized that a lower morphine dose (80 mcg, used regularly for this surgery in our hospital), would decrease postoperative opioid-related complications in hip arthroplasty while maintaining an excellent quality of anesthesia and analgesia. 


\section{Methods}

This study was approved by IRB of Eduardo Schütz Schroeder (Puerto Montt) Hospital and written informed consent was obtained from all patients. This manuscript adheres to the STROBE guidelines[6]. Our data collection process respected the privacy and anonymity of patients according to the Law of Rights and Duties of Patients established by the government of Chile ${ }^{7}$. This research did not receive any specific grant from funding agencies in the public, commercial, or not-for-profit sectors.

\section{Study design}

This was a case-control study. All patients undergoing total hip arthroplasty between 01/02/15 and 12/31/15 at Puerto Montt Hospital were recruited to participate. Inclusion criteria were: elective total hip replacement, age $\geq 18$ years, ASA $\leq 3$ and consented to the anesthetic protocol. Exclusion criteria were: polytrauma, allergy to any of the indicated medications, history of prior cerebral vascular accident with functional sequelae, depression, substance abuse or chronic opioid use. Patient who were known to need intensive care postoperatively, or did not complete the anesthetic protocol, were excluded from analysis. The comparative control group was identified retrospectively from patients treated at our hospital during the same time period but received $100 \mathrm{mcg}$ ITM. We also compared patients from two previously published reference works[1],[3].

\section{Anesthetic protocol}

After explanation of the anesthetic plan and informed consent, the patient was placed on standard American Society of Anesthesiologists (ASA) monitors and spinal anesthesia was performed in the sitting or lateral position at the L3-L4 level (or L2-L3 in case of technical difficulty) with $80 \mathrm{mcg}$ intrathecal morphine plus $1.4-1.8 \mathrm{ml}(10.5-13.5 \mathrm{mg})$ of hyperbaric bupivacaine $0.75 \%$, injected through a 25 Gauge (G) Whitacre spinal needle. Following administration of the spinal, the patient was placed in Trendelenburg position, and dermatomal level was measured every minute using a pinprick sensation testing with the tip of a $21 \mathrm{G}$ needle. Once a T8 - T10 anesthetic level was reached, the patient was returned to the neutral supine position. At ten minutes after the spinal, the dermatomal level was measured again to ensure adequate sensory coverage, and the patient was placed in the lateral decubitus position for surgery.
All patients received $40 \%$ oxygen via face mask. All patients received tranexamic acid $1 \mathrm{~g}$, cefazolin $2 \mathrm{~g}$ and dexamethasone $4 \mathrm{mg}$ intravenous (IV) prior to incision. Midazolam $0.1 \mathrm{mg} \cdot \mathrm{kg}^{-1} \mathrm{IV}$ was given PRN for intraoperative sedation. Mean arterial pressure (MAP) was maintained at $\geq 70 \mathrm{mmHg}$ by administering ringer's lactate and/or hydroxyethyl starch solution, and boluses of $100 \mathrm{mcg}$ phenylephrine or 12 mg ephedrine PRN at the discretion of the anesthesiologist. At the conclusion of the surgery, all patients observated in the post anesthesia care unit (PACU) by medical and nursing staff. Postoperative analgesia included dipyrone $4 \mathrm{~g}$ IV for 24 hours at rate $166 \mathrm{mg} /$ hr (or ketorolac $90 \mathrm{mg}$ at rate $3.75 \mathrm{mg} / \mathrm{h}$ in case of allergy) and paracetamol $15 \mathrm{mg} \cdot \mathrm{kg}^{-1}$. PO q $6 \mathrm{~h}$ until discharge. Pain was assessed using the visual analog scale (VAS) at 0, 6, 12, 24 and $48 \mathrm{~h}$ postoperatively and at discharge. If $V A S \geq 3$, morphine $0.05 \mathrm{mg} \mathrm{kg}^{-1}$ IV was administered for breakthrough pain. If pain persisted, one single dose of ketamine $0.2 \mathrm{mg} \cdot \mathrm{kg}^{-1} \mathrm{IV}$ was administrated.

Patients were assessed hourly for signs of complications, including respiratory depression (respiratory rate < 10 per minute), pruritus (patient report), urinary retention (absence of spontaneous diuresis after 6 hours), hypotension, (systolic blood pressure $<90 \mathrm{mmHg}$ or greater than $40 \%$ decrease from baseline) and bradycardia (heart rate $<60$ per minute or $<$ 40 per minute with chronic beta blocker usage).

Patients were transferred to the floor when motor and sensory blockade completely resolved in bilateral lower extremities (Bromage scale 4), pain was wellcontrolled (VAS $\leq 3$ ), and showed no sign of complications. In the case of respiratory depression, naloxone $0.4 \mathrm{mg} \cdot \mathrm{kg}^{-1} \mathrm{IV}$ was given if necessary. In the case of urinary retention, a single shot straight catheter was inserted. Hypotension was managed with crystalloids and boluses of phenylephrine 100 mcg or ephedrine $12 \mathrm{mg}$ IV. For nausea and/or pruritus, droperidol 0.625-1.25 mg IV was given, and if persisted after 30 minutes, a dose of ondansetron 4 mg IV was administrated.

\section{Data collection and outcome measures}

We recorded demographic variables such as age, sex, co-morbidities such as diabetes mellitus (DM), high blood pressure (HBP), chronic airflow limitation $(C A L)$, chronic kidney disease (based on serum creatinine level), stroke, active cardiac conditions as defined by the 2009 American Heart Association (AHA) guidelines[8], ASA classification and the use of pain medicine at baseline. Surgical variables, including in- 
dication, type of endoprosthesis, surgical time, time elapsed until mobilization, and total length of stay were also recorded.

Anesthetic variables included the dose of hyperbaric bupivacaine $0.75 \%$, dermatomal blockade level, recovery time according to the Bromage scale, the need for conversion to general anesthesia, pre and post anesthesia mean blood pressure (MAP), and need for vasoactive drugs. The primary outcome measures were analgesic quality according to VAS at 0,6 , 12,24 , and 48 hours. The secondary outcome measures were dose and frequency of rescue pain medicine needed, patient satisfaction prior to discharge indicated by a numeric Likert-like scale ranging from 1 (bad) to 10 (excellent), length of stay, any postoperative complication and 30-day mortality.

In an effort to address potential sources of bias, the data collection done by personnel not directly involved in the study design and blinded to the treatment group.

\section{Statistical analysis}

Data analysis was performed using Stata/MP $13^{\circledR}$ (Data Analysis and Statistical Software). Descriptive statistics, e.g., percentages and means, were provided. Student's t-test were used for parametric data between two groups, analysis of variance (ANOVA with post hoc Bonferroni correction) were used for comparison between multiple groups, and Chi-square analysis and odds ratios were used for comparison of proportions. Statistical significant was established at $p<0.01$. Sample size was calculated with Epidat 4.2, considering expected odds ratio 2.3, exposed proportion 70\% (ITM $80 \mathrm{mcg}$ ), controls proportion (ITM $100 \mathrm{mcg}), 1$ control per case, confidence interval (CI) 95\% and precision 5\%. Considering previous data, the required sample size was 12.794, per each group, a number that only large multicentric studies could achieve, because of that, we decide to use the sample of a full year for analysis.

\section{Results}

Eighty-two patients received the low dose $80 \mathrm{mcg}$ ITM and 66 patients received the conventional dose $100 \mathrm{mcg}$ ITM (Figure 1). There were no statistical difference in demographic and clinical background between the two groups, which were also similar to the retrospective control groups from Murphy and Slappendel's studies, as seen in data available from the previous publications (Table 1).
In our 80 mcg ITM, the mean age was 64.21 ( \pm $14.59)$ years, mean weight $74( \pm 10.25) \mathrm{kg}$, mean height $170( \pm 8.35) \mathrm{cm}, 62.20 \%$ were women and $70.73 \%$ were ASA II mostly due to a history of hypertension. The surgical time was similar between low ITM and control group, as well as retrospectively cited works, with mean time 71.30 ( \pm 21.21$)$ minutes. We observed a $15 \%$ decrease in mean blood pressure (MAP) following spinal anesthesia from pre-op MAP of $94.89 \mathrm{mmHg}$, and a $16.41 \%$ decrease in the hematrocit (Hct) at $24 \mathrm{~h}$ post-op from mean pre-op Hct of $38.70 \%$.

The main indications for hip arthroplasty were primary arthrosis (58.53\%), dysplasia (25.60\%), infection (8.53\%), and periprosthetic fracture (7.31\%). The subgroups based on pathology had statistically significant differences in demographics, including age, ASA status, HBP, stroke and serum creatinine. There were also significant differences in intraoperative bleeding, post-spinal MAP, postoperative hematocrit and the need for blood transfusions. Patients with arthroplasty for dysplasia had the lowest postspinal MAP but did not require more vasopressors. Patients with periprosthetic fracture appeared to be the sickest, with older average age, higher prevalence of atrial fibrillation and stroke, higher creatinine level, more intraoperative bleeding and need for blood transfusions, and lower postoperative Hct $(p<0.01)$. Interestingly, the same subgroup also required the lowest dose of intrathecal bupivacaine to get to the target anesthetic level. There was no significant difference in in surgical times among the subgroups.

Satisfactory anesthetic level of T9-T10 was obtained in all patients and there was no conversion to general anesthesia. The mean hyperbaric bupivacaine $(0.75 \%)$ dose was $11.55 \mathrm{mg}$, which was similar in both low-ITM and conventional ITM groups, but much lower than the retrospective controls from published data.

Postoperatively, $95.13 \%$ of patients reported VAS-0 during the first 48 hours, some remained painfree up to discharge. There was no statistically significant difference in pain scores between the pathology subgroups at any point. Three patients in the osteoarthritis group, and 1 in the fracture group, reported VAS-5 at 6-12 hours postoperative. In our control group, 3 patients reported VAS-5 at 6-12 hours postoperative (Figure 2). All patients were supplemented with IV morphine according to the protocol, averaging $3 \mathrm{mg}$ per patient ( $80 \mathrm{mcg}$ group) in the first 24 hours, statistically similar to our control group and Murphy's, but lower than Slappendel et al. No additional analgesics were required. 
Regarding postoperative complications associated with the use of intrathecal morphine (Figure 3, Table 1), in the low-ITM 80 mcg group, 2 patients presented with respiratory depression, in contrast to 4 patients in the $100 \mathrm{mcg}$ control group (odds ratio OR 2.58, confidence interval CI 95\% 0.45 - 14.54, $\mathrm{p}=0.28$ ). Nausea was reported in 14 low-ITM and 18 control patients (OR 1.82, Cl 95\% 0.82 - 4.01, p $=0.13$ ). No vomiting was reported. All episodes of PONV occurred during PACU stay and responded appropriately to droperidol. Urinary retention was seen in 12 low-ITM and 17 control patients, all resolved by a single bladder catheterization (OR 2.02, CI 95\% $0.88-4.61, p=0.09$ ). The most significant difference in adverse reactions was seen in the rate of pruritus, affecting 12 low-ITM and 25 control patients (OR 3.55, Cl 95\% $1.61-7.82, p<0.01)$. Seven low-ITM patients need blood transfusions, so did four control patients (OR 0.69, Cl 95\% $0.19-2.47, \mathrm{p}=0.56$ ). There was no significant difference in complications among the pathology subgroups (Table 1).

On average, PACU stay was 244.63 minutes and the total length of stay was 2.93 days. All of the patients had early mobilization with physical therapy within 24 hours of surgery. No one required postoperative ICU admission because of complications and there was no 30-day mortality. Mean rank patient satisfaction (focused in pain management) in the lowITM 80 mcg group was 8.57 (out of 10 points), statistically similar to the 100 mcg control group (mean rank 8.77).

\section{Discussion}

Many studies have searched for the ideal dose of intrathecal morphine for patients undergoing hip arthroplasty, mainly through a trial-and-error method[1]-[5]. Landmark articles by Slappendel and Murphy over a decade ago narrowed the dosage to 50-100 mcg[1],[3]. However, clinical disparity persists likely due to patient heterogeniety (extremes of age, comorbidities), diverse procedural coverage, new surgical and diagnostic techniques with variable surgical times, experiences of the treatment teams and lack of standarized perioperative protocol[5],[9].

Generally, $100 \mathrm{mcg}$ of intrathecal morphine is considered ideal for analgesia, but has a higher incidence of adverse events than $50 \mathrm{mcg}[1],[3]-[5]$. We hypothesized that an intermediate dosage of $80 \mathrm{mcg}$ would produce both: adequate analgesia and fewer adverse effects. This current study confirmed our hypothesis. No patient in our sample required conver- sion to general anesthesia. Pain scores were generally low. Minimal rescue IV medications were needed. In addition, patients reported high satisfaction levels for their pain management experience.

Comparative analysis with concurrent and retrospective control groups (receiving 100 to $50 \mathrm{mcg}$ of intrathecal morphine) with similar demographic and clinical characteristics demonstrates non-inferiority of our low-ITM regimen. Of note, the duration of surgery reported by Murphy was greater than ours (no data reported by Slappendel), with higher volume of intraoperative bleeding ${ }^{1}$. This may reflect the experience level of the team, pathology, surgical technique, or resources of the institutions when the original papers were published.

\section{Pain}

Extrapolating data from the two reference works, it seems that doses of 25-50 mcg of intrathecal morphine had lower incidence of complications but also delivered lower postoperative analgesia (mean VAS 1.92 during the first 24 hours)[1],[3]-[4],[11],[15]. Inadequate pain control may paradoxically increase the risk of respiratory depression due to administration of higher doses of rescue opioids such as morphine[10]-[12]. Conversely, 100-200 mcg of intrathecal morphine in the same studies, provided excellent analgesia (mean VAS 1.25 for the first 24 hours) but had higher incidence of adverse effects. Interestinly, our pain score with 80 mcg was even lower (mean VAS 0.25 , Figure 2). This may be related to clearer understand of pain mechanisms and application of multimodal analgesia and improvements in surgical technique.

We then examined how different ITM doses impacted inmediate and subsequent postoperative pain and recovery. We believe implementation of an active pain management protocol has an important role. Reassuringly, vast majority of our patients had no pain up to discharge.

Murphy reported that $60 \%$ of patients who received $50 \mathrm{mcg}$ of intrathecal morphine required analgesic supplementation postoperatively, averaging $12.5 \mathrm{mg}$ of IV morphine during the first 24 hours; whereas $26.6 \%$ of those who received 100 mcg required supplementation and the average dose was 3 mg of IV morphine, similar to our control group but much lower than Slappendel's 100 mcg group. The difference may be explained by the protocol management limits used in their respective works. Our patients had superior pain control, with just $4.87 \%$ receiving an average $3 \mathrm{mg}$ of IV morphine $(p<0.01)$. 


\section{Associated complications}

Respiratory depression is the most feared complication of systemic and intrathecal opioids (especially morphine)[10]. Our low-ITM group had 2 cases of respiratory compromise as determined by respiratory rate $(<10$ breaths per minute), compared to 4 patients in the control group. Although the small numbers preclude definitive conclusions, it seems that the association is exponential, doubling the risk with a small increasing in ITM dose $(80$ to $100 \mathrm{mcg}$, OR 2.58). Previous studies on hip arthroplasty with ITM reported incidences of respiratory depression ranging from $2.85 \%(25 \mathrm{mcg})$ to $10.8 \%(100 \mathrm{mcg})$, and none leading to mortality[1],[3]-[5],[15],[19],[20],[22]. In other contexts, such as elective cesarean section, when intrathecal morphine is administered in a single dose of $100-250 \mathrm{mcg}$, respiratory depression was observed to be $0.01-7 \%[14]$. The lack of an universally accepted definition (oximetry, respiratory rate, etc.) [13], differences in study duration and follow-up, and the confounding roles of other respiratory depressants such as benzodiazepines, may explain the discrepancies in results[1],[3].

Other potential complications such as PONV, urinary retention and pruritus, are also known contribute to morbidity and mortality and prolonged hospital stay (Figure 3)[12]. Our incidence of PONV $(17.07 \%)$ was slightly higher in control compared to low-ITM groups (OR 1.82), and overall much lower than the patients in previous publications. Direct relationship between the dose of intrathecal morphine and the prevalence of nausea and vomiting had been reported, reaching a "ceiling effect" of $50-60 \%$ of patients at 100-200 mcg[1],[3],[11],[15],[16]. We recorded nausea and/or emesis as a single variable, and relied on patient report or visual detection by nurses for distinguish the two symptoms. This may have contributed to a lower reported incidence rate. Of note, $35 \%$ of our patients received antiemetic therapy per protocol, similar to other studies[1],[3],[16].

Urinary retention is more frequently observed in men following spinal anesthesia (ratio 2:1). Regardless of the diagnostic criteria, there is a direct correlation between the dose of intrathecal morphine and the presence of urinary retention[1],[3],[17]. In our study $14.65 \%$ (80 $\mathrm{mcg}$ ) versus $25.75 \%$ (100 mcg control group) experienced this complication (OR 2.02), far below the average $40 \%$ reported in other series[1],[3],[17]. All of the instances of urinary retention were self-limited and resolved within 24 hours, with none or at most one episode of straight urinary catheterization.
We diagnosed pruritus by direct patient report, similar to Murphy's and Slappendel's studies[1],[3]. This is the most frequently reported side effect associated with intrathecal opioids[1],[3],[19]. Given its unclear pathophysiological mechanism in neuraxial anesthesia[18], there seem to be an exponential association between the dose of intrathecal morphine and incidence and severity of pruritus, ranging from $20 \%$ (50 mcg), to $38.6 \%(100 \mathrm{mcg})$ and $70 \%$ (200 mcg) [1],[3]. In our sample, the OR for developing pruritus was 3.55 with the higher $100 \mathrm{mcg}$ ITM vs $80 \mathrm{mcg}$, confirmed to be statistically significant. Residual sedation may have limited the patient's ability to verbalize, and antiemetics (droperidol) may prophylactically treated pruritus[16],[18]. We suspect that more proactive questioning by nurses may have yielded more responses.

We also emphasize that the $80 \mathrm{mcg}$ group presented a lower requirement for blood products (OR 0.69) than the control group, however this was not statistically significant and may be an incidental finding that is explained by surgical factors rather than pharmacological or the anesthetic technique.

\section{Functional recovery and satisfaction}

All patients started ambulation with physical therapy within 24 hours after surgery, and met the criteria of free walking with orthoses before discharge. There were no statistically significant differences between the groups in average length of stay (2.93 versus 2.88 days). Patient satisfaction between our groups was also similar, sadly, we could not find any comparable data about this aspect from previously studies.

\section{Limitations and benefits}

Not being a clinical trial per se was the main limitation of our study. We consider this a "mixed study" that attempted to replicate a known intervention using a reduced medication dosage in a similar population, matched to demographic and clinical characteristics of the existing studies (when available) and concurrent controls. Even though we did not randomise the treatment groups prospectively, the patients were matched doe demographic and clinical backgrounds and the outcomes assessments were conducted blindly by provders not involved in the trial deisgn. Our advantage was the relatively large sample size and detailed follow up. Unfortunately, we could not get the original data from previously published studies, limiting our comparison analysis with other works.

We must also consider that advances in technol- 
ogy and medical education may have translated into an improved standard of care today in contrast with ten years ago, confounding our comparison with the previous studies.

Caution is warranteed when generalizing our results (and those of other series consulted) to more complex patients at the extremes of age, ASA > 3, or with cardiovascular comorbidities. These groups have not been adequately represented in any of the studies on hip arthroplasty and should be explored futher[2],[21]. For future research, we propose a prospective randomized controlled trial examining different reduced dosages of intrathecal morphine, with longer duration of follow-up to evaluate the longterm recovery results, and determine what to prioritize in clinical applications.

\section{Conclusions}

Our results confirmed that spinal anesthesia with a reduced dose of $80 \mathrm{mcg}$ intrathecal morphine provides adequate anesthesia and postoperative analgesia for patients undergoing hip arthroplasty, with therapeutic effects comparable to 100 - $200 \mathrm{mcg}$ ITM. This dose also resulted in lower incidence of adverse effects, especially pruritus, at rates comparable to $50 \mathrm{mcg}$ intrathecal morphine[22]. Standardization of perioperative protocol and early detection of associated complications likely contributed the positive outcomes we observed. Continued training of healthcare professional's application of new technologies would help optimize our methods with the goal to promote patient comfort, safety and rapid recovery.

\section{Acknowledgements}

We thank Qing Yang MD, PhD, Massachusetts General Hospital, Department of Anesthesia, Critical Care and Pain Medicine, Harvard Medical School, Boston, Massachusetts, United States, for critically reviewed the proposal.

\section{References}

1. Murphy PM, Stack D, Kinirons B, Laffey JG. Optimizing the dose of intrathecal morphine in older patients undergoing hip arthroplasty. Anesth Analg. 2003;97:1709-1715.

2. Foadi $N$, Karst M, Frese-Gaul A, Rahe-Meyer N, Krömer S, Weilbach $C$. The improved quality of postoperative analgesia after intrathecal morphine does not result in improved recovery and quality of life in the first 6 months after orthopedic surgery: a randomized controlled pilot study. J Pain Res. 2017;9:10591069. doi:10.2147/JPR.S135142

3. Slappendel R, Weber E, Dirksen R, Gielen MJ, van Limbeek J. Optimization of the dose of intrathecal morphine in total hip surgery: a dose finding study. Anesth Analg. 1999;88:822-826.

4. Højer-Karlsen AP, Geisler A, Petersen PL, Mathiesen O, Dahl
$J B$. Postoperative pain treatment after total hip arthroplasty: a systematic review. Pain. 2015;156:8-30. doi:10.1016/j. pain.0000000000000003

5. Kuchálik J, Granath B, Ljunggren A, Magnuson A, Lundin A, Gupta A. Postoperative pain relief after total hip arthroplasty: a randomized, doubleblind comparison between intrathecal morphine and local infiltration analgesia. Br J Anaesth. 2013;111:793-799. doi: 10.1093/bja/aet248.

6. von Elm E, Altman D, Egger $M$, Pocock S, Go P, Vandenbroucke J. The Strengthening the Reporting of Observational Studies in Epidemiology [STROBE] statement: guidelines for reporting observational studies. Gac Sanit. 2008;22:144-150.

7. Patient's rights and duties. Chilean Health Ministry. Available from http://web.minsal. cl/derechos-y-deberes-de-los- pacientes/ Published October 1, 2012. [Accessed 23 Juny 2017]

8. Fleisher LA, Beckman JA, Brown KA y cols. 2009 ACCF/AHA focused update on perioperative beta blockade incorporated into the ACC/AHA 2007 guidelines on perioperative cardiovascular evaluation and care for noncardiac surgery: a report of the American College of Cardiology Foundation/ American Heart Association Task Force on Practice Guidelines. Circulation. 2009;120:e169e276. doi:10.1161/CIRCULATIONAHA.109.192690.

9. Fenten MG, Keijzer BA, Stienstra R. No clinically relevant advantage of intrathecal morphine in total hip arthroplasty? Acta Anaesthesiol Belg. 2014;65:161166.

10. Bailey PL, Lu JK, Pace NL y cols. Effects of intrathecal morphine on the ventilatory response to hypoxia. $\mathrm{N}$ 
Engl J Med. 2000;343:1228-

1234. doi:10.1056/

NEJM200010263431705

11. Gehling M, Tryba M. Risks and side-effects of intrathecal morphine combined with spinal anaesthesia: a meta-analysis. Anaesthesia. 2009;64:643-651

12. Thompson MJ, Clinger BN, Simonds RM, Hochheimer CJ, Lahaye LA, Golladay GJ. Probability of undiagnosed obstructive sleep apnea does not correlate with adverse pulmonary events nor length of stay in hip and knee arthroplasty using intrathecal opioid. J Arthroplasty. 2017;14:161-164 doi:10.1016/j. arth.2017.02.043.

13. Horlocker TT, Burton AW, Connis RT y cols. American Society of Anesthesiologists Task Force on Neuraxial Opioids. Practice guidelines for the prevention, detection, and management of respiratory depression associated with neuraxial opioid administration. Anesthesiology. 2009;110:218-230 doi:10.1097/ ALN.0b013e31818ec946.

14. Dalchow S, Lubeigt O, Peters G,
Harvey A, Duggan T, Binning A. Transcutaneous carbon dioxide levels and oxygen saturation following caesarean section performed under spinal anaesthesia with intrathecal opioids. Int J Obstet Anesth. 2013;22:217-222. doi:10.1016/j.ijoa.2013.04.003.

15. Domsky M, Kwartowitz J. Efficacy of subarachnoid morphine in a community hospital. Reg Anesth. 1992;17:279-282.

16. Koyama S, Kurita S, Nakatani K, Nagata N, Toyoda Y. Preoperative intravenous administration of droperidol (1.25 mg) reduced postoperative nausea and vomiting after intrathecal morphine administration. Masui. 2011;60:173-179.

17. Griesdale DE, Neufeld J, Dhillon D y cols. Risk factors for urinary retention after hip or knee replacement: a cohort study. Can J Anaesth. 2011;58:1097-1104. doi:10.1007/s12630-011-9595952.

18. Brull R, Macfarlane A, Chan V. Spinal, Epidural and Caudal Anesthesia. In: Miller R, ed. Anesthesia, 8th Edition. Philadelphia,
Elsevier, 2015;1684-1717.

19. Yamashita K, Fukusaki M, Ando Y, Tanabe T, Terao Y, Sumikawa K. Postoperative analgesia with minidose intrathecal morphine for bipolar hip prosthesis in extremely elderly patients. J Anesth. 2009;23:504-507. doi:10.1007/s00540-009-08175.

20. Kwan AS, Lee BB, Brake $T$. Intrathecal morphine for postoperative analgesia in patients with fractured hips. Hong Kong Med J. 1997;3:250-255.

21. Macfarlane AJ, Prasad GA, Chan VW, Brull R. Does regional anaesthesia improve outcome after total hip arthroplasty? A systematic review. Br J Anaesth. 2009;103:335-345. doi:10.1093/ bja/aep208.

22. OMendieta Sánchez JM, Fernández-Liesa Jl, Marcos G, Panadero A, Sánchez-Leedesma, Macías A. Efficacy of $0.1 \mathrm{mg}$ of subarachnoid morphine combined with bupivacaine on postoperative analgesia in total hip arthroplasty. Rev Esp Anestesiol Reanim. 1999;46:433-437 